\title{
MODELING OF BEAM-IONIZED SOURCES FOR PLASMA ACCELERATORS
}

\author{
S. Deng ${ }^{1}$, C. D. Barnes ${ }^{3}$, C. E.Clayton ${ }^{2}$, C. O’Connell ${ }^{3}$, F. J. Decker ${ }^{3}$, P. Emma ${ }^{3}$, O. Erdem ${ }^{1}$, C.

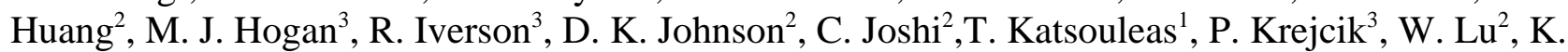

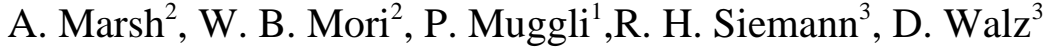 \\ ${ }^{1}$ University of Southern California, Los Angeles, CA 90089 \\ ${ }^{2}$ University of California, Los Angeles, CA 90095 \\ ${ }^{3}$ Stanford Linear Accelerator Center Stanford, CA 94309
}

\begin{abstract}
When considering intense particle or laser beams propagating in dense plasma or gas, ionization plays an important role. Impact ionization and tunnel ionization may create new plasma electrons, altering the physics of wakefield accelerators, creating and modifying instabilities, etc. Here we describe the addition of an ionization package into the 3-D object-oriented fully parallel PIC code OSIRIS [1]. Using intense beams to tunnel-ionize neutral gas may become a new source of plasma. For the beams whose electrical fields are right above threshold, the optimal gas density for maximize electrical field is about $7 \mathrm{n}_{0}\left(\mathrm{n}_{0}\right.$ is the optimal density according to linear theory $\left.\omega_{\mathrm{p}} \sigma_{z} / \mathrm{c}=2^{1 / 2}[2]\right)$. We apply the simulation tool to the parameters of the current E164 [3] Plasma Wakefield Accelerator experiment at the Stanford Linear Accelerator Center (SLAC). We find that tunnel ionization affects the wakefield and energy gain of E-164 experiment.
\end{abstract}

\section{INTRODUCTION}

Following the success of recent plasma-based accelerators (peak accelerating gradients on the order of $200 \mathrm{MeV} / \mathrm{m}$ for E157/E162 [4]), experiments are underway to achieve yet higher gradients with shorter and more intense drive beams. For these, ionization the working gas by the beam becomes important. In future experiments and concepts such as E164X and the afterburner, a high-density short bunch is used to drive nonlinear (blowout regime) plasma wakes, and multi-GeV peak accelerating gradients are achieved. The need for long homogeneous plasma sources of high density (up to 10 meters of $2 * 10^{16} \mathrm{~cm}^{-3}$ plasma for the afterburner) makes the possibility of a plasma self-ionized by the drive beam attractive.

In this paper, we study the optimal gas density at which the plasma wakefield is maximized for a self-ionized plasma wakefield accelerator. Simulation results for the E164 experiment and E164X experiment are presented.

\section{PIC CODE OSIRIS AND IONIZATION MODELS}

OSIRIS is a fully relativistic PIC code with newly added ionization package which includes 2D and 3D impact ionization and tunnel ionization.
The probability [6] for a particle to impact-ionize the gas is

$$
\mathrm{P}_{\mathrm{i}}=\operatorname{ng} \sigma\left(\mathrm{v}_{\mathrm{i}}\right)\left|\mathrm{v}_{\mathrm{i}}\right| \Delta \mathrm{t}
$$

Here $n_{g}$ is gas density, $\sigma$ : is gas cross-section, and $\mathrm{v}$ is the velocity of the incident particle.

For tunnel ionization, the Ammosov-DeloneKrainov(ADK)[7] model is used to calculate the tunneling ionization probability. According to the ADK formula, the ionization rate for $\mathrm{Li}$ is give by the following equation:

$$
\omega(\mathrm{t})=\Delta \mathrm{t}^{*} 3.46^{*} 10^{21} \exp (-85.5 / \mathrm{E}) / \mathrm{E}^{2.18}
$$

Here $\mathrm{E}$ is the amplitude of the applied electrical field, with unit $\mathrm{GV} / \mathrm{m}$.

OSIRIS is an object-oriented program. It enables us to add an ionization module without changing other existing modules. The ionization module can interact with other modules freely.

\section{IONIZATION AS A NEW PLASMA SOURCE}

. Using short bunch with high density to tunnel-ionize neutral gas is a possible way to create plasma. In Fig. 1, newly created electrons are pushed away by the driving beam and form a wake.

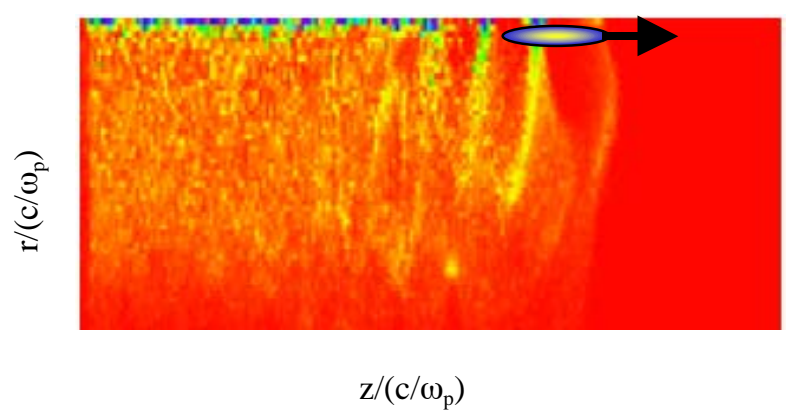

Fig 1. Real space of new electrons

We did two runs to check the amplitude of the wakefield: run 1 is for a short beam with electric fields far above threshold, and run 2 is for a beam with electric field just above threshold. 
The physical parameters are shown in table 1 .

Table 1: physical parameters

\begin{tabular}{|l|l|l|}
\hline & Run 1 & Run 2 \\
\hline $\begin{array}{l}\text { Beam Energy } \\
(\mathrm{GeV})\end{array}$ & 30 & 50 \\
\hline $\mathrm{N}$ & $2 \mathrm{e} 10$ & $2 \mathrm{e} 10$ \\
\hline$\sigma_{\mathrm{z}}(\mu \mathrm{m})$ & 20 & 63 \\
\hline$\sigma_{\mathrm{r}}(\mu \mathrm{m})$ & 20 & 14.1 \\
\hline $\mathrm{n}_{0}\left(\mathrm{~cm}^{-3}\right)$ & $4.41 * 10^{16}$ & $1.4 * 10^{16}$ \\
\hline $\mathrm{n}_{\mathrm{gas}}\left(\mathrm{cm}^{-3}\right)$ & $1.25^{-3} 10^{17}$ & $4.2^{*} 10^{16}$ \\
\hline
\end{tabular}

$\mathrm{n}_{0}$ is the optimal density according to linear theory $\omega_{\mathrm{p}} \sigma_{z} / \mathrm{c}=2^{1 / 2}$.

In both case, $\mathrm{n}_{\text {gas }}$ is around 3 times $\mathrm{n}_{0}$. As one sees in Fig. 2, the wakefield created in the self-ionization case is comparable to that in the pre-ionization case for far above threshold case --case 1(the pre-ionized plasma density is set to equal to gas density). For the case just above threshold --case 2 (pre-ionized plasma density is set to equal to $\mathrm{n}_{0}$ ) the wakefields are smaller. This is because for a beam with field just above threshold, the head of the beam cannot ionize gas until its electric field reaches the threshold value. The rapidly ionized plasma then "sees" an effectively shortened beam, because it does not see the head of the beam. A higher gas density is needed to match the plasma period (wavelength) to the effective pulse length.

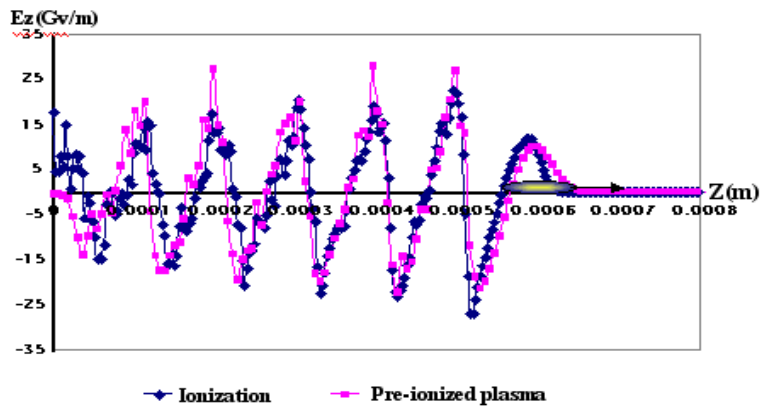

Fig 2a). Comparison of longitudinal $E$ field of preionized case and self-ionized cases for run 1

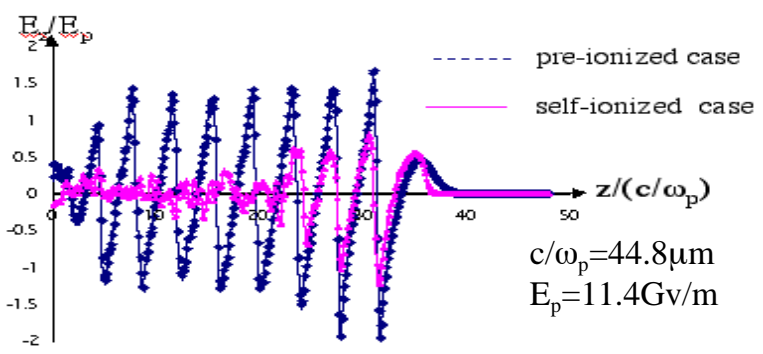

Fig 2b). Comparison of longitudinal E field of preionized case and self-ionized cases for run 2
Parameter search results to find the optimal gas density at which the wakefield are maximized for run 2 are shown in Fig 3. As we expected, the electrical fields peak at higher density $\left(7 \mathrm{n}_{0}\right)$ for self-ionied case than pre-ionized case $\left(3 n_{0}\right)$. Even in the pre-ionized case, the optimal density is larger than the linear theory optimal density $n_{o}$ because the non-linear wake drives the plasma electrons relativistically, increasing their mass and decreasing the plasma frequency. The density needs be higher to compensate this frequency decrease.

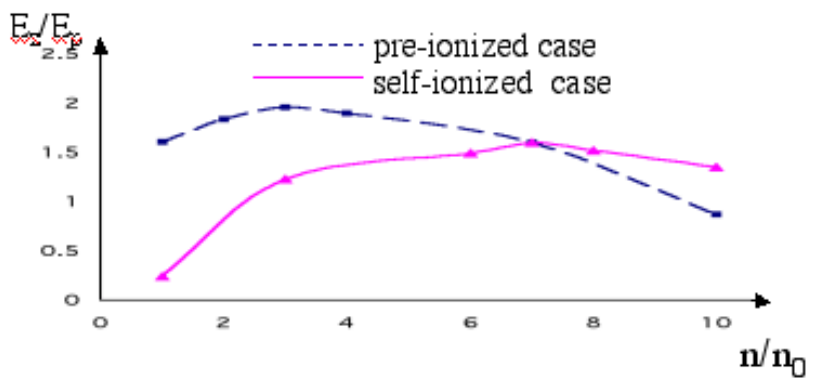

Fig 3. Peak longitudinal E field vs. gas or plasma density

\section{SIMULATION FOR E 164}

3 comparison runs are done for E164 parameters. Case 1 is for with both pre-ionized plasma and tunnel-ionized plasma. Case 2 is for pre-ionized plasma only. Case 3 is for tunnel-ionzed plasma only. The physics parameters are shown in table 2 .

Table 2: physical parameters for e164 runs

\begin{tabular}{|l|l|l|l|}
\hline & Case 1 & Case 2 & Case 3 \\
\hline $\begin{array}{l}\text { Beam Energy } \\
(\mathrm{GeV})\end{array}$ & 30 & 30 & 30 \\
\hline $\mathrm{N}$ & $2 \mathrm{e} 10$ & $2 \mathrm{e} 10$ & $2 \mathrm{e} 10$ \\
\hline$\sigma_{\mathrm{z}}(\mu \mathrm{m})$ & 100 & 100 & 100 \\
\hline$\sigma_{\mathrm{r}}(\mu \mathrm{m})$ & 25 & 25 & 12.5 \\
\hline $\mathrm{N}_{\text {plasma }}\left(\mathrm{cm}^{-3}\right)$ & $5^{*} 10^{15}$ & $5^{*} 10^{15}$ & 0 \\
\hline $\mathrm{N}_{\text {gas }}\left(\mathrm{cm}^{-3}\right)$ & $4.5^{*} 10^{16}$ & 0 & $2.5^{*} 10^{16}$ \\
\hline
\end{tabular}

Fig. 4 shows the Energy gain and the lineout of longitudinal electrical wakefield for each case. The increased plasma density due to new created electrons makes the wakefield wavelength shorter (see the comparision diagram for case 1 and case 2. For E164 parameters, the wakefield are close to threshold of tunnel ionisation; the magnitude of wakefield and energy gain for case 3 is much less than the pre-ionized case. 


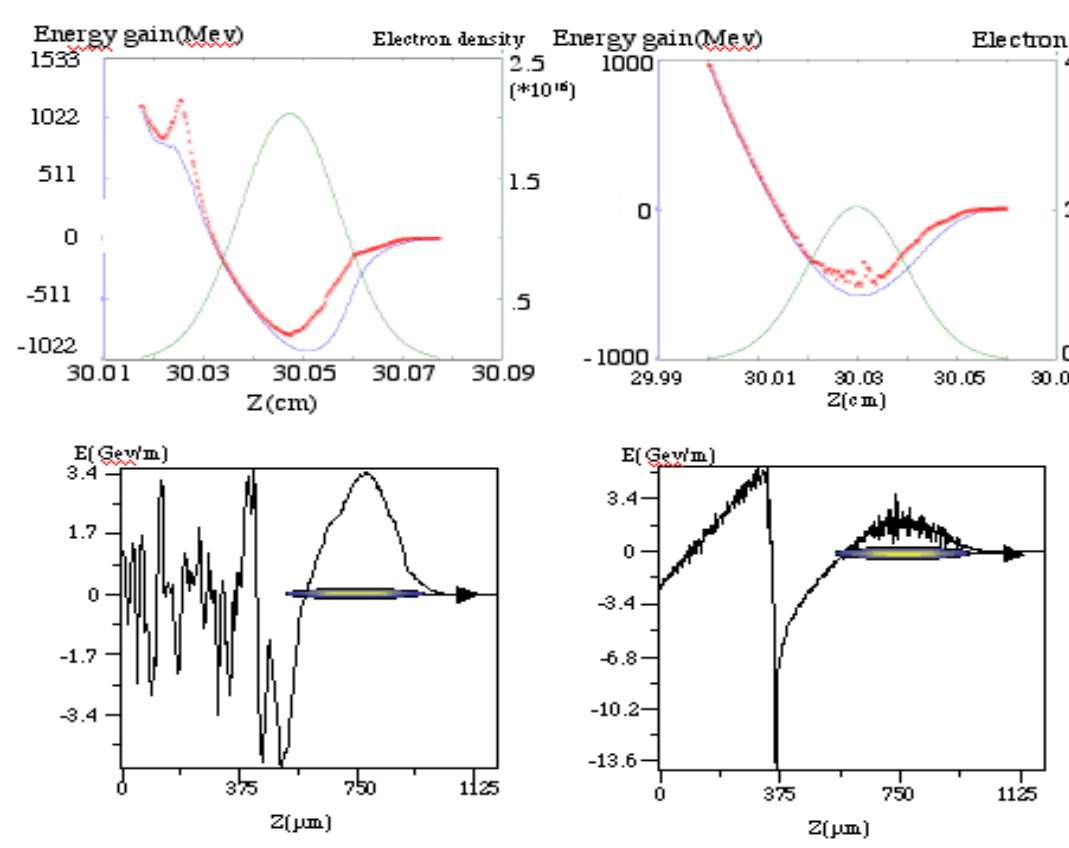

Case 1: pre-ionized and tunnel-ionized Case 2: pre-ionized plasma only
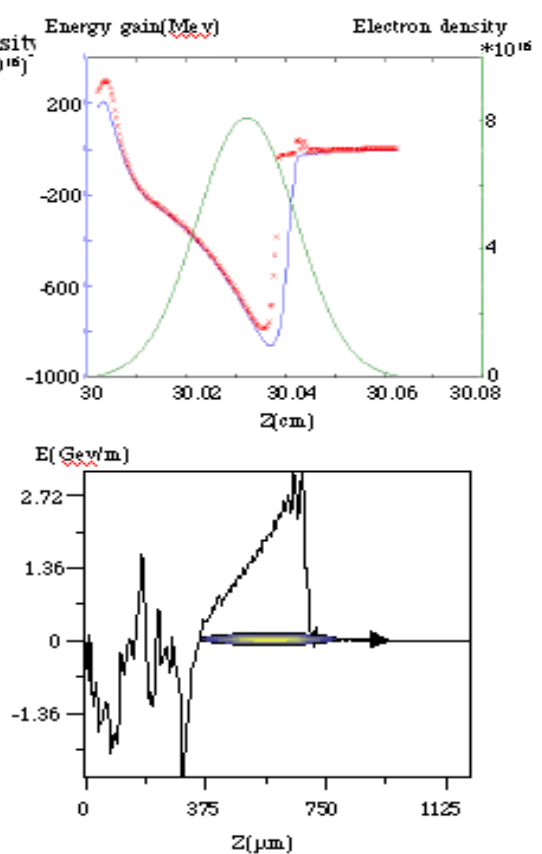

Case 3: tunnel ionised plasma only

Fig 4. Comparision results for E164 (x's represent maximum energy gain, blue line represents average gain; The Gaussian shows the beam profile)

\section{SIMULATION FOR E164X}

In E164X, even shorter beams (10-20 $\mu \mathrm{m})$ are used than in E164 $(100 \mu \mathrm{m})$. In this case, the electrical field of the beam is far beyond the threshold. As one can see in Fig, 5, $25 \mathrm{GeV} / \mathrm{m}$ peak acceleration fields can be achieved, and the peak average energy gain is around $25 \mathrm{MeV}$ for a $2 \mathrm{~mm}$ run. These results support the thesis that self- ionization can be used as a way to create plasma sources for plasma wakefield accelerators and the beam wakefield can be made comparable to the pre-ionized plasma case.
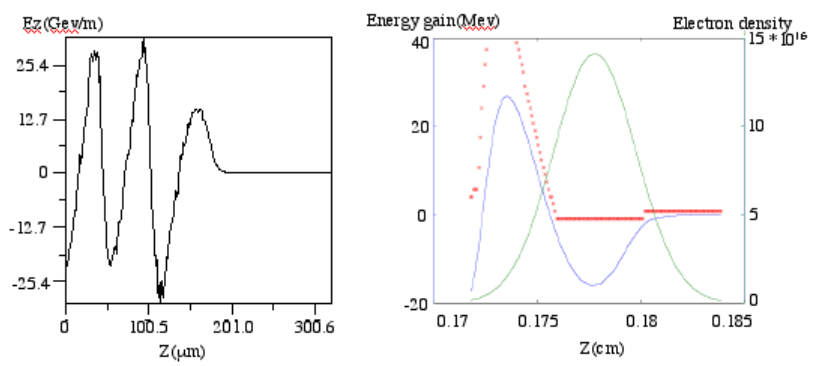

Fig 5. E164X simulation results

\section{CONCLUSION}

Tunnel ionization cannot be neglected when very short beams are driving the plasma wakefield. The new created electron can shorter the wavelength and yield similar or smaller wakefields, depending on how short the beam is. For very short beams (like E164X), the wakefields are comparable to the pre-ionzed case, while for beams whose fields are near threshold (like E164), higher density is required to optimally match. More work will be done to study the optimal density.

\section{ACKNOWLEDGMENTS}

The authors gratefully acknowledge helpful conversations with David L. Bruhwiler. This work is supported by U.S. Department of Energy, under Contracts No. DE-FG03-92ER40745,DE-FC02-01ER41192, DEFC02-01ER41179, DE-FG03-92ER40727, and NSF PHY-0078508.

\section{REFERENCES}

[1] R. G. Hemker, F. S. Tsung, V. K. Decyk, W. B. Mori, S. Lee, and T..Katsouleas, Development of a parallel code for modeling plasma based accelerators, IEEE Particle Accelerator Conference 5 , 3672-3674 (1999).

[2] E164 proposal, unpublished.

[3] R. Keinigs and M.E. Jones, Phys. Fluids 30, 252 (1987)

[4] David L. Bruhwiler, Rodolfo E. Giacone, Physical Review Special Topics, vol. 4, pp. 101302 (2001)

[5] E164X proposal, unpublished.

[6] V. Vahedi and M. Surendra, Comput. Phys. Commun. 87, 199(1995)

[7] M.V. Ammosov, N.B. Delone and V.P.Krainov, Sov. Phys. JETP 64, 1191 (1986) 